\title{
CONSTRUCCIÓN Y VALIDACIÓN DE UN INSTRUMENTO PARA MEDIR CALIDAD DE ATENCIÓN DEL PROGRAMA AUGE CÁNCER CERVICOUTERINO *
}

\author{
María Teresa Urrutia S., $P h D^{1 a}$, Lauren Poupin B., MSc ${ }^{a}$ \\ ${ }^{1}$ Escuela de Enfermería, Facultad de Medicina, Pontificia Universidad Católica de Chile. \\ a Enfermera Matrona
}

* Proyecto financiado por el Fondo Nacional de Investigación en Salud, CONICYT-MINSAL, CHILE. Proyecto FONIS SA05I20047.

\section{RESUMEN}

Antecedentes: La evaluación de la calidad de atención, integrando la opinión de los usuarios, es uno de los parámetros a considerar al momento de evaluar los servicios en salud. No existen instrumentos validados a nivel nacional que permitan evaluar la calidad de la atención en patologías del programa AUGE. Objetivos: Crear y validar un instrumento para medir calidad de atención en el programa AUGE cáncer cérvicouterino. Método: El análisis fue realizado en un grupo de 364 mujeres pertenecientes al programa. Para la validación del instrumento se utilizó análisis factorial exploratorio, y la confiabilidad fue medida por alpha de Cronbach. Resultados: El instrumento final denominado INCA-AUGE presenta 19 ítems distribuidos en 5 dominios: funcionamiento del programa AUGE, relación usuaria-profesional, infraestructura, recurso humano, educación sobre la patología AUGE, y recursos materiales. Conclusión: El instrumento INCA-AUGE pretende ser una herramienta útil en su aplicación, asegurando una medición válida de los parámetros para recoger objetivamente la evaluación de las usuarias del programa.

\section{PALABRAS CLAVE: Cáncer cérvicouterino, calidad de atención, programa AUGE}

\section{SUMMARY}

Background: Quality of care evaluation, considering the opinion of the users, is one of the parameters to consider at the time of evaluating the services in health. Objectives: Because the absence of validated and reliable national questionnaires, this article describes the development and testing of a new questionnaire to measure quality of care in cervical cancer AUGE program. Method: The analysis was done in a group of 364 women. Exploratory factor analysis was used to validate the questionnaire and Cronbach alpha to measure reliability. Results: The final instrument named INCA-AUGE has 19 items with 5 domains: operation of AUGE program, relationship healthcare worker- patient, infrastructure and human resource, education on AUGE disease, and material resources. Conclusion: INCA-AUGE questionnaire will be a useful tool, assuring a validated and reliable measurement on the patients of AUGE program.

\section{KEY WORDS: Cervical cancer, quality of care, AUGE program}




\section{INTRODUCCIÓN}

El avance de las enfermedades crónicas, para las cuales no existe una curación total ha llevado a proponer tratamientos que consisten básicamente en atenuar o eliminar síntomas. Esto ha revelado que las medidas clásicas de resultados en salud no sean suficientes para evaluar la calidad de los servicios (1). La evaluación de la calidad de atención (CA) es uno de los parámetros a considerar al momento de evaluar los servicios en salud (2), señalando que la opinión de las usuarias es un indicador prioritario para este objetivo $(3,4)$.

Las usuarias valoran la atención rápida en casos de urgencia y la posibilidad de recibir servicios preventivos en salud (5). Se señala que un gran porcentaje de las mujeres quiere recibir consejería al momento del diagnóstico y apoyo a lo largo del tratamiento $(6,7,8)$, hecho demostrado por la insatisfacción con la educación recibida $(7,8,9)$. Las usuarias valoran ser vistas por el mismo profesional, ser referidas a centros de especialidad y citadas a controles sólo en casos necesarios $(3,5)$; señalan que los profesionales no les explican lo que ellas necesitan (10), aludiendo una falta de sensibilidad, de continuidad en el cuidado, barreras en el lenguaje y largos tiempos de espera (8).

Como en el proceso de atención en salud se comprometen tanto aquellos que brindan el cuidado como quienes lo reciben, los aspectos señalados por las usuarias debieran compararse y de manera especial complementarse con la visión que los profesionales tienen, para entregar un cuidado más apropiado $(3,5)$. Se ha visto que la perspectiva de los usuarios no es necesariamente la misma que la de los profesionales en lo referente a CA (11).

Los profesionales opinan que la falta de tiempo es una barrera que se interpone para poder crear la adherencia de las pacientes (8), señalando que la información entregada a la usuaria sobre el diagnóstico es el aspecto más relevante a considerar, así como también sobre el progreso de la enfermedad, el tratamiento, el pronóstico y el lugar donde recibirán el cuidado (12).

En nuestro país, desde el año 2004 se encuentra en vigencia el programa AUGE (Acceso Universal a Garantías Explícitas), y una de las patologías garantizadas es el cáncer cérvicouterino (CC). No existen publicaciones que reporten la evaluación de la CA desde la perspectiva de las usuarias del programa AUGE-CC. Por otro lado se pudo comprobar que no existen instrumentos que permitan medir la calidad de atención del programa AUGE-CC. El objetivo de este artículo es describir la creación y validación de un instrumento para medir $\mathrm{CA}$ en el programa AUGE-CC.

\section{MATERIAL Y MÉTODO}

Características de la muestra en estudio: La recolección de los datos se realizó en el periodo AbrilOctubre del 2006. Los criterios de inclusión fueron haber ingresado al programa AUGE-CC del Servicio de Salud Metropolitano Sur Oriente (SSMSO) en los años 2004 o 2005 y tener diagnóstico confirmado por biopsia de lesión invasora o pre-invasora. Un total de 364 mujeres ingresaron al estudio, de las cuales 127 mujeres tienen CC y 237 lesiones pre-invasoras. Las 127 mujeres corresponden al universo de mujeres vivas con CC ingresadas al programa; se decidió acceder al universo completo de mujeres con $\mathrm{CC}$ dado que son las únicas que entregar información acerca de la CA recibida en los tres niveles de atención. Las 237 mujeres con lesiones pre-invasoras corresponden a una muestra representativa con un $95 \%$ de confianza, de la población de mujeres con lesiones pre-invasoras del programa AUGE-CC del SSMSO. El promedio de edad de las mujeres es de 42,2 $\pm 11,9$ años (rango: 22 - 81 años). La escolaridad promedio de las mujeres es de 9,03 $\pm 0,72$ años de estudio. Las características de la muestra en estudio se presentan en la Tabla I. El estudio fue aprobado por los comités de ética de la Escuela de Enfermería de la Pontificia Universidad Católica de Chile y del SSMSO. Se solicitó firmar consentimiento informado a cada una de las mujeres participantes.

\section{Tabla I}

\section{CARACTERÍSTICAS DE LAS MUJERES DEL ESTUDIO}

\begin{tabular}{llrr}
\hline Variable & & $\mathrm{n}$ & $\%$ \\
\hline Año de ingreso & 2004 & 141 & 38,7 \\
al programa & 2005 & 221 & 60,7 \\
AUGE & & & \\
Hospital de & Doctor Sótero del Rio & 207 & 56,9 \\
pertenencia & Padre Alberto Hurtado & 157 & 43,1 \\
& & & \\
Previsión & FONASA A & 188 & 51,6 \\
& FONASA B & 100 & 27,5 \\
& FONASA C & 47 & 12,9 \\
& FONASA D & 24 & 6,6 \\
& PRAIS & 4 & 1,1 \\
& No Sabe & 1 & 1 \\
& Analfabeta & 8 & 2,2 \\
Ultimo nivel & Básica & 140 & 38,5 \\
de escolaridad & Media & 191 & 52,5 \\
aprobado & Técnica & 12 & 3,3 \\
& Universitaria & 11 & 3,0 \\
\hline
\end{tabular}

FONASA: Fondo Nacional de Salud. PRAIS: Programa de Reparación y Atención Integral de Salud y Derechos Humanos: AUGE: Acceso Universal a Garantías Explícitas. 
Características del instrumento: El instrumento original propone 26 ítems distribuidos en 10 dominios (Tabla II). Todos los ítems están dirigidos a evaluar la CA recibida por las mujeres en el programa AUGE-CC. Para la elaboración de los ítems se recurrió a una revisión de la literatura cualitativa y cuantitativa en el área, junto con entrevistas a expertos nacionales. Una vez elaborado el instrumento, se procedió a realizar la validez de contenido con 3 profesionales expertos en el área de CA y/o gestión de servicios en salud. La validación por expertos no arrojó cambios en el instrumento propuesto. Posterior a la validez de contenido, se realizó una prueba piloto en 15 mujeres para comprobar que las preguntas se entendieran y calcular el tiempo de aplicación del instrumento. Hubo solo cambios de redacción en algunos de los ítems. La medición de los ítems se realizó a través de escalas de Likert de 3 alternativas (si / parcialmente / no), de 5 alternativas (muy buena / buena/ regular /mala / muy mala o siempre / casi siempre/ a veces/ casi nunca / nunca) y una escala de calificación del 1 al 7.

\section{Tabla II}

ÍTEMS Y DOMINIOS RESPECTIVOS DEL INSTRUMENTO INICIAL

\begin{tabular}{lll}
\hline Dominio & Ítem & Escala de Likert
\end{tabular}

Continuidad en la atención profesional

Seguridad

Infraestructura

Recursos del programa

Relación Usuaria Profesional

Vías de comunicación

Capacidad resolutiva
1. En los diferentes servicios, las matronas, médicos y enfermeras que la atendieron por esta enfermedad ¿fueron siempre los mismos?

2. Para usted ¿es importante que sea siempre el mismo profesional el que la atienda?

3. En los diferentes servicios, las matronas, médicos y enfermeras que la atendieron por esta enfermedad ¿le daban confianza?

4. En los diferentes servicios, las matronas, médicos y enfermeras que la atendieron por esta enfermedad ¿le brindaron la privacidad necesaria?

5. En los diferentes servicios donde fue atendida ¿cuentan con la infraestructura necesaria para brindar una atención satisfactoria a las usuarias?

6. En los diferentes servicios donde fue atendida ¿eran cómodos los lugares donde esperaba?

7. En los diferentes servicios donde fue atendida ¿tenían los materiales necesarios para brindarle una buena atención?

8. En los diferentes servicios donde fue atendida el material utilizado ¿estaba en buenas condiciones para realizar la atención?

9. En los diferentes servicios donde fue atendida ¿es suficiente la cantidad de personal que atiende a las usuarias?

10. En los diferentes servicios donde fue atendida ¿siente usted que le brindaban un cuidado humanizado?

11. En los diferentes servicios donde fue atendida ¿usted se atrevía a hacerles preguntas cuando no entendía alguna explicación o cuando deseaba saber algo más?

12. En los diferentes servicios donde fue atendida ¿cómo diría que fue la comunicación que establecieron con usted?

13. En los diferentes servicios donde fue atendida ¿siente usted que le daban solución a los problemas que usted tenía debido a su enfermedad?
Si / Parcialmente / No

Si / Parcialmente / No

Si / Parcialmente / No

Si / Parcialmente / No

Si / Parcialmente / No

Si / Parcialmente / No

Si / Parcialmente / No

Si / Parcialmente / No

Si / Parcialmente / No

Si / Parcialmente / No

Si / Parcialmente / No

Muy Buena / Buena/ Regular / Mala / Muy Mala

Siempre / Casi siempre / A veces / Casi Nunca /Nunca 
14. En los diferentes servicios donde fue atendida, cuándo usted tenía que ir de un servicio a otro ¿le daban explicaciones de cómo y cuándo acudir?

15. ¿Tuvo citaciones que no se cumplieron, es decir la citaron y no la atendieron?

16. En los diferentes servicios donde fue atendida ¿la espera para ser atendida era larga?

Calidad profesional

Diagnóstico y tratamiento de la enfermedad

Evaluación general del Programa
17. En los diferentes servicios donde fue atendida los profesionales ¿se identificaban por su nombre?

18. En los diferentes servicios donde fue atendida cuando los profesionales le daban alguna información ¿se tomaban el tiempo para asegurarse que usted comprendía?

19. En los diferentes servicios donde fue atendida los profesionales ¿le daban opción a preguntar o a expresar sus inquietudes?

20. En los diferentes servicios donde fue atendida los profesionales que la atendieron ¿la nombraban a usted por su nombre?

21. Considerando los diferentes servicios donde fue atendida ¿qué nota le pondría a los profesionales que la atendieron durante su enfermedad?

22. ¿Le explicaron en qué consistía su enfermedad (diagnóstico)?

23. ¿Le explicaron en qué consistía el tratamiento que debía seguir?

24. Considerando todos los aspectos anteriormente evaluados ¿qué nota le pondría usted a la atención recibida en este programa (AUGE-CC)?

25. ¿Recomendaría usted a otra persona, familiar o amiga con esta enfermedad, atenderse en este programa en este mismo servicio de salud?

26. ¿Hizo algún reclamo por escrito alguna vez?
Si / Parcialmente / No

$\mathrm{Si} / \mathrm{No}$

Si / Parcialmente / No

Si / Parcialmente / No

Si / Parcialmente / No

Si / Parcialmente / No

Si / Parcialmente / No

Escala de nota del 1 al 7

Si / Parcialmente / No

Si / Parcialmente / No

Escala de nota del 1 al 7

Si / Parcialmente / No

Si / No
El instrumento elaborado fue aplicado por matronas y enfermeras-matronas entrenadas previamente para ese propósito. El entrenamiento consideró la explicación de cada uno de los ítems y las alternativas de respuestas, junto a un ejercicio de role-playing para valorar las habilidades previamente explicadas.

Evaluación psicométrica: La validez del instrumento fue medida a través de análisis factorial exploratorio (AFE) y la confiabilidad por medio del alpha de Cronbach. En el AFE se utilizó la factorización de ejes principales y para la rotación de factores, se eligió la rotación ortogonal-Varimax. Los criterios de selección de los ítems y de los factores fueron: autovalores menores de 1 (método de Kaiser y Catell), porcentaje de varianza explicada, 20 más ítems por factor, valor del coeficiente factorial mayor de 0,3, presencia de coeficientes factoriales en más de un factor (diferencia menor de 0,15 ) y finalmente la interpretación conceptual del ítem en relación al factor seleccionado.

\section{RESULTADOS}

Distribución normal: El rango de curtosis de los ítems del instrumento propuesto, varía entre 0,097 y 13,96 con 2 ítems con valores mayores a 2,03. El rango de asimetría fue de 0,27 y 3,86. Dado que el porcentaje de curtosis fue menos del $8 \%$ del total de los ítems, los 26 ítems ingresaron al AFE (13). Matriz de correlación: El análisis de la matriz de correlación de los ítems del instrumento es el primer paso en el AFE (14). Las mediciones de adecuación de muestra señalan la aplicabilidad del análisis factorial. El test Kaiser-Meyer-Olkin (KMO) señala un valor de 0,840 , considerado meritorio, señalando que existe una muestra satisfactoria en relación al número de ítems en el cuestionario. El test de 
Bartlett presenta un valor $x^{2}=2101, p<0,001$, lo que señala que existe relación entre los diferentes ítems.

Análisis factorial exploratorio: Cuatro modelos fueron calculados hasta lograr el modelo definitivo. El primer modelo fue calculado con 26 ítems. De acuerdo al criterio de Kaiser, 8 factores son retenidos explicando el $39,57 \%$ de la varianza. Las matrices de factores rotados señalan 4 ítems con coeficientes factoriales menores de 0,3 , los cuales fueron eliminados (ítems 1, 11, 15 y 16). El segundo modelo fue calculado con 22 ítems. De acuerdo al criterio de Kaiser, 6 factores son retenidos explicando el $40,08 \%$ de la varianza. Los ítems con un coeficiente factorial menor de 0,3 fueron eliminados (items 2 y 26). El tercer modelo fue calculado con 20 ítems. De acuerdo al criterio de Kaiser, 5 factores son retenidos explicando el $41,7 \%$ de la varianza. El ítem 4 fue el único ítem con un coeficiente factorial menor de 0,3 el cual fue eliminado.

El último modelo calculado fue con 19 ítems. De acuerdo al criterio de Kaiser, 5 factores son retenidos explicando el $42,81 \%$ de la varianza. No hubo ítems con coeficientes factoriales menores de 0,3 por lo que se decide retener este modelo como modelo final. En la Figura 1 se presenta el gráfico de sedimentación y en la Tabla III la matriz factorial del modelo final.

El nuevo instrumento denominado INCA-AUGE (Instrumento Calidad de Atención- AUGE) considera 19 ítems distribuidos en 5 dimensiones (Tabla IV): funcionamiento del programa (6 ítems); relación usuaria-profesional (6 ítems); infraestructura y recurso humano (3 ítems); educación sobre la patología AUGE (2 ítems) y recursos materiales (2 ítems).

Confiabilidad del instrumento: El valor del alpha de Cronbach en el instrumento INCA- AUGE fue de 0,70 en este grupo de estudio.

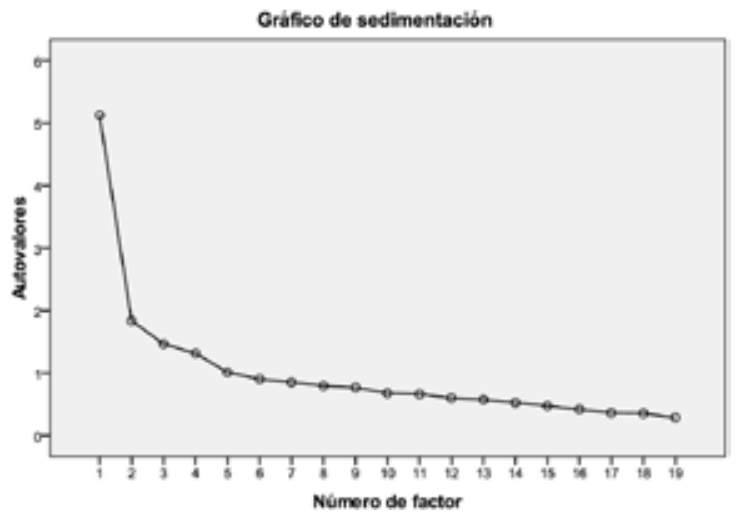

Figura 1. Gráfico de sedimentación del modelo final.

\section{DISCUSIÓN}

El CC como patología ha demostrado ser un importante trazador para el estudio de la CA en salud, ya que involucra variadas etapas y proveedores, presentándose como un continuo desde el nivel primario al terciario. En cada uno de estos niveles se pueden presentar barreras, fortalezas y debilidades en el proceso, los que al ser evaluados pueden develar los errores y las potencialidades para establecer medidas de calidad (15).

Cabe señalar que las 5 dimensiones consideradas en el INCA-AUGE, apuntan a aspectos centrales al momento de brindar una atención de calidad. En relación a la dimensión funcionamiento del programa AUGE, ésta se presenta como un área con aspectos generales a considerar. Uno de ellos es la calificación que las usuarias hacen del programa y sus profesionales, lo que refleja el grado de satisfacción global con la atención recibida, aspecto que ha sido descrito previamente como un determinante de la CA (16). Otro aspecto considerado en esta primera dimensión y que también coincide con publicaciones previas (16), es la capacidad que tiene el programa de dar solución a los problemas de las usuarias producto de su enfermedad. Respecto del ítem de comunicación, se ha descrito que

Tabla III

\section{MATRIZ FACTORIAL MODELO FINAL INCA-AUGE}

\begin{tabular}{|c|c|c|c|c|c|}
\hline \multirow[b]{2}{*}{ ITEM } & \multicolumn{5}{|c|}{ Factores } \\
\hline & 1 & 2 & 3 & 4 & 5 \\
\hline Ítem 21 & $-0,724$ & & & & \\
\hline Ítem 24 & $-0,721$ & & & & \\
\hline Ítem 13 & 0,613 & & & & \\
\hline Ítem 12 & 0,488 & & & & \\
\hline Ítem 25 & 0,476 & & & & \\
\hline Ítem 3 & 0,367 & & & & \\
\hline Ítem 17 & & 0,779 & & & \\
\hline Ítem 19 & & 0,680 & & & \\
\hline Ítem 17 & & 0,465 & & & \\
\hline Ítem 10 & 0,382 & 0,438 & & & \\
\hline Ítem 14 & 0,303 & 0,354 & & & \\
\hline Ítem 20 & & 0,334 & & & \\
\hline Ítem 6 & & & 0,592 & & \\
\hline Ítem 5 & & & 0,571 & & \\
\hline Ítem 9 & & & 0,466 & & \\
\hline Ítem 23 & & & & 0,813 & \\
\hline Ítem 22 & & & & 0,743 & \\
\hline Ítem 7 & & & 0,334 & & 0,678 \\
\hline Ítem 8 & & & & & 0,496 \\
\hline
\end{tabular}

INCA-AUGE: Instrumento Calidad de Atención-AUGE. 
la CA se verá favorecida en la medida que la comunicación establecida por el profesional sea efectiva y respetuosa con la usuaria (17). Las mujeres desean una comunicación cercana, uno a uno, para lo cual el tiempo que el profesional entregue y la confianza brindada son claves para lograr una mejor satisfacción (17). Finalmente la recomendación o no de este programa a una amiga o familiar es otro aspecto incluido en esta dimensión, y que ha sido considerado por otros autores al momento de evaluar CA (18). Es importante señalar que la adherencia a programas de salud en $\mathrm{CC}$ ha sido relacionada con la evaluación que hace la mujer del sistema de salud y de la CA brindada $(19,20)$. Por lo tanto, considerar el mejoramiento de la CA podría implicar en nuestro país una mayor adherencia al programa en sus diferentes niveles de atención.

Tabla IV

ÍTEMS Y DOMINIOS RESPECTIVOS DEL INSTRUMENTO INCA-AUGE

\begin{tabular}{lll}
\hline Dominio & Ítem & Escala de Likert \\
\hline
\end{tabular}

\section{Dimensión I:}

Funcionamiento del programa AUGE

Dimensión II:

Relación usuaria-profesional
1. Considerando los diferentes servicios donde fue atendida ¿qué nota le pondría a los profesionales que la atendieron durante su enfermedad?

2. Considerando todos los aspectos anteriormente evaluados ¿qué nota le pondría usted a la atención recibida en este programa?(CC-AUGE)

3. En los diferentes servicios donde fue atendida ¿siente usted que le daban solución a los problemas que usted tenía debido a su enfermedad?

4. En los diferentes servicios donde fue atendida ¿cómo diría que fue la comunicación que establecieron con usted?

5. ¿Recomendaría usted a otra persona, familiar o amiga con esta enfermedad, atenderse en este programa en este mismo Servicio de Salud?

6. En los diferentes servicios, las matronas, médicos y enfermeras que la atendieron por esta enfermedad ¿le daban confianza?

1. En los diferentes servicios donde fue atendida los profesionales ¿se identificaban por su nombre?

2. En los diferentes servicios donde fue atendida cuando los profesionales le daban alguna información ¿se tomaban el tiempo para asegurarse que usted comprendía?

3. En los diferentes servicios donde fue atendida los profesionales ¿le daban opción a preguntar o a expresar sus inquietudes?

4. En los diferentes servicios donde fue atendida los profesionales que la atendieron ¿la nombraban a usted por su nombre?

5. En los diferentes servicios donde fue atendida ¿siente usted que los profesionales le brindaban un cuidado humanizado?

6. En los diferentes servicios donde fue atendida, cuándo usted tenía que ir de un servicio a otro ¿los profesionales le daban explicaciones de cómo y cuándo acudir?

\section{Escala de nota}

del 1 al 7

Escala de nota

del 1 al 7

Siempre / Casi siempre

A veces / Casi Nunca /Nunca

Muy Buena / Buena / Regular

Mala / Muy Mala

$\mathrm{Si} /$ Parcialmente / No

Si / Parcialmente / No

Si / Parcialmente / No

Si / Parcialmente / No

Si / Parcialmente / No

Si / Parcialmente / No

Si / Parcialmente / No

Si / Parcialmente / No 
Dimensión III:

Infraestructura y recurso humano

Dimensión IV:

Educación sobre la patología AUGE

Dimensión V: recursos materiales
1. En los diferentes servicios donde fue atendida ¿cuentan con la infraestructura necesaria para brindar una atención satisfactoria a las usuarias?

2. En los diferentes servicios donde fue atendida ¿eran cómodos los lugares donde esperaba?

3. En los diferentes servicios donde fue atendida ¿es suficiente la cantidad de personal que atiende a las usuarias?

1. ¿Le explicaron en qué consistía su enfermedad (diagnóstico)?

2. ¿Le explicaron en qué consistía el tratamiento que debía seguir?

1. En los diferentes servicios donde fue atendida ¿tenían los materiales necesarios para brindarle una buena atención?

2. En los diferentes servicios donde fue atendida el material utilizado ¿estaba en buenas condiciones para realizar la atención?
Si / Parcialmente / No

Si / Parcialmente / No

Si / Parcialmente / No

Si / Parcialmente / No

Si / Parcialmente / No

Si / Parcialmente / No

Si / Parcialmente / No
La segunda dimensión identificada en INCA-AUGE fue la relación usuaria-profesional donde aspectos básicos, como que el profesional se identifique por su nombre y a su vez identifique a la usuaria, ingresaron a la dimensión, también han sido descritos previamente como características de una buena CA (17). La entrega de información por parte del profesional, asegurándose que la usuaria entienda, y dándole espacio para expresar alguna duda, fueron 3 de los 6 aspectos considerados en este dominio, todos ellos respaldados en la literatura como parte de la CA (17). La falta de tiempo ha sido considerada una barrera para que las mujeres se atrevan a preguntar, por lo que la percepción para que el profesional le de espacio a hacer preguntas es valorado como parte de una buena atención (17).

Cabe destacar que la calificación de un cuidado humanizado, es una característica que también fue considerada como parte de la relación usuaria-profesional de calidad; al respecto Thind y cols (16), señalan que las mujeres reportan altos niveles de satisfacción con la atención recibida cuando reciben un mayor apoyo emocional por parte de los profesionales. La relación usuaria profesional ha sido descrita como un área importante de la CA $(17,21)$, e incluso ha sido también descrita como predictor de la calidad de vida de mujeres con cáncer ginecológico (22). Por lo tanto esta dimensión en el INCA-AUGE cobra gran relevancia en lo que respecta a evaluar dicha interacción y también para relacionarla con resultados en salud (17).

Respecto de la dimensión infraestructura y recursos humanos, tanto la calidad del lugar donde son atendidas como la cantidad de personal fueron considerados en esta dimensión. Específicamente importa la comodidad de los sitios en los cuales las mujeres deben esperar atención profesional. En un estudio multicéntrico, se reportó que tanto la apariencia como la limpieza de los lugares de atención son aspectos importantes a considerar por las mujeres para la evaluación de la CA recibida (17). Junto a esto, el espacio disponible (18) y la privacidad que se establezca en las salas de espera, minimizando el número de personas, ha sido considerado como una variable importante a considerar (17). Este aspecto estaría directamente relacionado al tiempo que deben esperar para ser atendidas (18).

Resulta relevante la importancia que logra la cuarta dimensión del INCA-AUGE que presenta altos coeficientes en la matriz factorial, y que hace referencia a la educación sobre la patología AUGE. La explicación de la enfermedad AUGE que la mujer padece como de su tratamiento son los 2 aspectos considerados en esta dimensión. Cabe señalar que la usuaria se encuentra vulnerable al momento de recibir el diagnóstico de cáncer, por lo que el apoyo brindado por parte del personal que la atiende es fundamental, siendo el profesional que entrega el diagnóstico el actor mas importante en dicha interacción $(16,22)$. La educación entregada a los usuarios ha sido considerada uno de los estándares de CA $(21,23)$. Al respecto cabe señalar que a mayor información recibida y a mayor participación en la toma de decisión respecto del tratamiento a recibir por parte de la usuaria, es mayor el grado de satisfacción con la atención recibida (16), siendo 
esto congruente con la aparición de esta dimensión como un dominio en el INCA-AUGE. Las usuarias desean que los profesionales les expliquen en un lenguaje simple y gentil, respetando la confidencialidad que este proceso implica $(17,21)$.

Los recursos materiales es la quinta dimensión que surge en el análisis factorial, con 2 ítems que dan cuenta por un lado si se tienen los materiales necesarios y por otro lado si esos materiales están en buenas condiciones. Es interesante destacar que los recursos materiales cobran una dimensión diferente a la infraestructura y recursos humanos, relevando esta dimensión como independiente de las otras. Estudios previos reportan la limpieza de los instrumentos y de la camilla de atención (17) como una característica importante a considerar evaluar la CA, al igual que la escasez de material (18).

El instrumento desarrollado, señala 5 dimensiones a considerar al momento de evaluar la CA de un programa AUGE-CC en Chile, resultando un producto de gran utilidad en el estudio de la CA en nuestro país, ya que ha sido validado en población chilena usuaria del sistema público de atención y representativa de un programa AUGE de uno de los Servicios de Salud del país.

El instrumento, al considerar aspectos generales del programa AUGE-CC, podrá ser utilizado en otros programas AUGE del país, lo que a su vez permitirá realizar evaluaciones de los mismos programas en diferentes periodos de tiempo y comparar la atención entre los diferentes programas.

La implementación de estrategias que permitan mejorar la CA exige conocer cuáles son los aspectos que requieren ser modificados antes de llevar a cabo nuevas intervenciones.

\section{CONCLUSIÓN}

El instrumento de medición de CA pretende ser una herramienta útil en su aplicación, asegurando una medición válida de los parámetros a evaluar, y permitiendo recoger la retroalimentación proveniente de uno de los principales protagonistas: las usuarias.

\section{BIBLIOGRAFÍA}

1. Schwartzmann L. Calidad de vida relacionada con la salud: Aspectos conceptuales. Cienc Enferm 2003;9:9-21.

2. Hulscher MEJL, Laurant MGH, Grol RPTM. Process evaluation on quality improvement interventions. Qual Saf Health Care 2003;12:40-6.

3. Wensing M, Mainz J, Ferreira $P$, Hearnshaw $H$, Hjortdahl P, Olesen F, Reis S, Ribacke M, Szécsényi J, Grol R. General practice care and patients' priorities in Europe: an international comparison. Health Policy 1998;45:175-86.

4. Larsson G, Larsson B, Munck I. Refinement of the questionnaire "quality of care from the patient's perspective using structural equation modelling. Scand $J$ Caring Sci 1998,12:111-8.

5. Wensing $M$, Jung $H$, Olesen $F$, Grol R. A systematic review of the literature on patient priorities for general practice care. Part 1: Description of the research domain. Sco Sci Med 1998;47:1573-88.

6. Wenzel L, DeAlba I, Habbal R, Coffey Kluhsman B, Fairclough D, U. Krebs L, Anton-Culver H, Berkowitz R, Aziz N. Quality of life in long-term cervical cancer survivors. Gynecol Oncol 2005;97:310-7.

7. Basen-Engquist K, Paskett E, Buzaglo J, Miller S, Schover L, Wenzel L, Badurka D. Cervical Cancer. Behavioral factors related to screening, diagnosis, and survivors' quality of life. Cancer 2003;98:2009-14.

8. Ashing-Giwa K, Kagawa-Singer M, Padilla G, Tejero J, Hsiao E, Chhabra R, Martínez L, Tucker MB. The impact of cervical cancer and dysplasia: A qualitative, multiethnic study. Psychooncology 2004;13:709-28.

9. Klee M, Groenvold M, Machin D. Using data from studies of health-related quality of life to describe clinical issues examples from a longitudinal study of patients with advanced stages of cervical cancer. Qual Life Res1999;8:733-42.

10. Juraskova I, Butow P, Robertson R, Sharpe L, McLeod C, Hacker N. Post-treatment sexual adjustment following cervical and endometrial cancer: A qualitative insight. Psychooncology 2003;12:267-79.

11. Hendriks A.A.J, Oort F.J, Vrielink M.R, Smets E.M.A. Reliability and validity of the satisfaction with hospital care questionnaire. Int J Qual Health Care 2002;14:471-82.

12. Arraras JI, Wright S, Greimel E, Holzner B, KuljanicVlasic K, Velikova G, Eisemann M, Visser A. Development of a questionnaire to evaluate the information needs of cancer patient: the EORTC questionnaire. Patient Educ Couns 2004;54:235-41.

13. Ferguson E, Cox, T. Exploratory factor analysis: A user's guide. Int J Selection Assess 1993;1:84-94.

14. De Vellis R. Factor analysis. En De Vellis R Editor. Scale development: Theory and applications; 2003. p. 102-137. Thousand Oaks: Sage Publications.

15. Zapka J, Puleo E, Taplin S, Goins K, Ulcickas M, Mouchawar J, Somkin C, Manos M. Processes of care in cervical and breast cancer screening and followup - the importance of communication. Prev Med 2004;39:81-90.

16. Thind A, Hog L, Diamant A, Maly RC. Satisfaction with care among low-income women with breast cancer. J Womens Health 2010;19:77-86.

17. Bingham A, Bishop A, Coffey P, Winkler J, Bradley J, Dzuba I, Agurto I. Factors affecting utilization of 
cervical cancer prevention services in low-resource setting. Salud Publica Mex 2003;45 suppl 3:S408-16.

18. Gomez-Jauregui J. Costos y calidad de la prueba de detección oportuna del cáncer cérvicouterino de una clínica pública y en una organización no gubernamental. Salud Publica Mex 2001;43:279-88.

19. Schmidt V, de Azevedo M, Galvão Ch, Carandina L,Goldbaum M. Fatores associados à não realização do exame de Papanicolaou: um estudo de base populacional no Município de Campinas, São Paulo, Brasil. Cad Saúde Pública 2006; 22:2329-38.

20. Agurto I, Arrosi S, White S, Coffey P, Czuba I, Bingham A, Lewis R. Involving the community in cervical cancer preventions programs. Int J Gynaecol Obstet 2005;89(supl 2):S38-45.
21. Tisheman C, Lundgren EL, Skald A, Tornberg S, Larsson BW. Quality of care from patient perspective in population-based cervical cancer screening. Acta Oncol 2002;41:253-61.

22. Awadalla A, Ohaeri J, Gholoum A, Khalid A, Hamad $\mathrm{H}$, Jacob A. Factors associated with quality of life of outpatients with breast cancer and gynecologic cancers and their family caregivers: a controlled study. BMC Cancer 2007;19:102-15.

23. Borenstein J, Badamgrav E, Henning JM, Gano $A D$, Weindarten SR. The association between quality improvement activities performed by managed care organizations and quality of care. Am J Med 2004;117:297-304. 